\title{
Lernen Online - Infrastruktur und Interaktion
}

*josef.strobl@sbg.ac.at, Interfakultärer Fachbereich Geoinformatik - ZGIS, Universität Salzburg

eingereicht am: 12.04.2020, akzeptiert am: 28.04.2020

Über viele Jahre hinweg wurden in nationalen und europäischen Projekten digitale Lernmaterialien und Lernmedien entwickelt. Waren wir auf dieser Grundlage gut auf den abrupten Wechsel hin zu E-Learning online vorbereitet? Weil ja gilt ,content is king', oder gehört vielleicht doch etwas mehr dazu, um Lernen in einer Internet-Umgebung erfolgreich zu gestalten? Die erforderlichen Kompetenzen bei Lehrenden und Lernenden inkludieren insbesondere auch interaktive Kommunikation und flexiblen wie auch kritischen Umgang mit digitalen Werkzeugen und dynamischen Medien. Darin liegen Aufgaben für zukünftige Unterrichtsgestaltung.

Keywords: E-Learning, Distance-Learning, IT-Infrastruktur, Lernen

\section{E-Learning - Infrastructure and Interaction}

Over many years, national and European projects supported the development of digital learning materials and media. Did this prepare us sufficiently for the unexpected switch towards e-learning? Beyond the 'content is king'-phrase - is there more to the successful implementation of learning in internet and cloud-based environments? The competences required from all stakeholders clearly focus on online interaction and collaboration skills, and on working with dynamic media. We have our work set for the redesign of learning in a digital world.

Keywords: e-learning, distance learning, IT-infrastructure, learning process

\section{Corona als Startpunkt}

In der aktuellen „Coronakrise“ wurde der Unterrichtsbetrieb an Österreichs Schulen, auch mangels Alternativen, spontan und wenig ausdifferenziert auf online „umgeschaltet“. Die dabei gemachten Erfahrungen, gewonnenen Einsichten und diagnostizierten Defizite werden in den nächsten Monaten und Jahren sicherlich aufgearbeitet werden und die Grundlage für weitere Entwicklungen in der ,Digitalisierung' von Lehren und Lernen bilden.

Derzeit ist es für empirisch fundierte und validierte Erkenntnisse wohl noch zu früh, allerdings ist es durchaus an der Zeit einen Rahmen für deren Gewinnung abzustecken. Der Brückenschlag von ad hoc gemachten Erfahrungen zu verallgemeinerbaren Prinzipien und deren Umsetzung in einen zukünftigen Rahmen für ,best practice' wird unterschiedliche Zugänge benötigen und wohl differenzierte Handlungsempfehlungen mit sich bringen.

Die Fächer Geographie und Geoinformatik an der Universität Salzburg haben über Jahrzehnte Erfahrung in Online-Fernstudien gesammelt. Manche dieser Erkenntnisse treffen wohl vorrangig für Erwachsene zu, allerdings war ein Faktum immer klar: Digitale Bereitstellung von Lernmaterialien und Medien reicht bei Weitem nicht für die Erreichung von Lernzielen aus. Der soziale und kommunikative Kontext eines Studiums ist ein vorrangiger Erfolgsfaktor. - Sonst bestände kein Unterschied zwischen Schule und Buchladen.

\section{Dann machen wir das halt online}

Für lange Zeit hatte E-Learning einen mäßig guten Ruf, war im schulischen wie im universitären Sektor nicht als Zugang erster Wahl akzeptiert und günstigenfalls in unterstützender Funktion vertretbar. Der plötzliche Sachzwang zu disloziertem Online-Betrieb führte zum gehäuften Versand von Arbeitsblättern, einer vielfach angebotsgesteuerten Nutzung von Online-Ressourcen und einer spontanen Nutzungsänderung informeller Online-Kommunikationskanäle.

Viele der unmittelbar gemachten Erfahrungen haben das Potenzial, die Akzeptanz von Online-Lernen 
nachhaltig zu schädigen. Soziale Medien, und in der Folge auch Massenmedien, sind voll von elterlichen Wut- und Verzweiflungs-Statements einerseits und von schulischen wie auch ministeriellen Diagnosen unterbrochener Kommunikation zu ,verlorenen' Schülerinnen und Schülern andererseits. Als kurzfristige Abhilfe wird mit Massenbeschaffung von Endgeräten reagiert. Wenngleich damit eine essenzielle Vorbedingung erfüllt wird, kann damit jedoch nicht die zugrundeliegende Problematik behoben werden.

Vordergründiger Auslöser der plötzlichen Legitimation des Online-Unterrichtens war wohl auch die Reaktion mancher Unternehmen auf, work-fromhome' umzustellen (das, glaubt man gängigen Statistiken, nur für ca. 20-30\% beruflicher Profile nachhaltig machbar ist). Lehrer/innen, als ,Wissensarbeiter/ innen' verstanden, können demnach auch ihren Job online erledigen. In Altersgruppen der Lernenden, wo Lehrer/innen vermehrt als Mentorinnen und Mentoren agieren und umfassendere Arbeitsaufträge erteilen, ist dies wohl auch realistisch, in jüngeren Altersgruppen aber absurd.

Warum? - In manchen Bildungssystemen gibt es die Option von ,home-schooling. Diese bedingt jedoch die Übernahme wesentlicher Teile der Rolle von Lehrerinnen und Lehrern durch andere Betreuungspersonen vor Ort. Wenn nun Eltern wegen eigener Telearbeit, anderer sozialer Aufgaben oder auch mangels Eignung bzw. Motivation diese Rolle nicht übernehmen können, wird eine reine Übermittlung von Lernmaterialien - mit oder ohne Angebote zur Hilfestellung und zur Zielkontrolle - nicht im Sinne der Lernziele funktionieren (können).

,Learning-from-home' kann daher in keiner Weise mit ,work-from-home' gleichgesetzt werden. Voraussetzungen und Rahmenbedingungen sind grundlegend anders. Die aktuellen, situationsbezogen gemachten Erfahrungen können und sollen daher zukunftsweisend eingesetzt werden, um digitale Komponenten in einer ganzheitlichen Unterrichtsgestaltung als Mehrwert und nicht als minderwertigen Ersatz zu positionieren.

\section{Terminologie}

Viele der Begriffe werden in einem breiten Verständnis synonym verwendet, einige sollen daher kurz eingegrenzt werden:

E-Learning bzw. computergestütztes Lernen - Erwerb von Wissen und Kompetenzen unter vorwiegender Nutzung digitaler Medien. Die reine elektronische Übermittlung von Materialien hat damit im Gegensatz zu gängiger Praxis nichts zu tun.
Online-Lernen - Vorrangiger Einsatz Internetbasierter interaktiver Lehr- und Lernmedien.

Virtuelles Klassenzimmer - Ein ,Ort' im Internet, der als Treffpunkt einer Gruppe (Klasse) dient. Die Verlinkung asynchron zu nutzenden Materialien reicht dafür nicht aus. Die sozial-kommunikative Komponente mit entsprechenden gemeinsamen synchronen Phasen muss wohl im Mittelpunkt stehen.

Tele-Teaching - Die lehrende Person steht typischerweise via Video im Mittelpunkt. Es ist zwischen aufgezeichneten Sequenzen und interaktiv-reaktiven (synchronen) VideokonferenzFormaten zu unterscheiden.

Diese Begriffe, auch im Kontrast zur häufig damit verbundenen Praxis, demonstrieren auch die Verwendung von Metaphern (wie ,Klassenzimmer'), die aber letztlich leider nicht als digitaler Zwilling der realen Welt eingerichtet werden. Der soziale Kontext eines Klassenzimmers ist ja doch mehr als eine OnlinePinnwand mit Link-Listen.

\section{Prinzip ,Cloud'}

Die grundlegenden Bausteine von digitalem bzw. Online-Lernen unterliegen auch einer stetigen Weiterentwicklung, die z. B. die Erfahrungen und Prinzipien der ersten Generation von E-Learning heute nur mehr begrenzt gültig macht.

Ein langsamer, aber stetiger Wandel der letzten Jahre betrifft die Infrastruktur des Internet selbst. Ursprünglich einfach als Verbindung zwischen einzelnen Rechner-Knoten konzipiert, dient es heute als Zugang zu generischen Online-Diensten mit vielfach unscharfer Wahrnehmung (daher auch, cloud computing'). Diese werden global verteilt betrieben, sind jedoch operativ hoch zentralisiert. - Man adressiert Google, Amazon, Facebook, Microsoft365 unabhängig vom eigenen Standort und auch dem der jeweiligen Rechenzentren.

Eine derart globalisierte Infrastruktur bringt enorme Vorteile wie Einheitlichkeit, Skalierbarkeit und Stabilität, unterliegt aber auch den Vorbehalten hinsichtlich Datenschutz, Kontrolle über eigene Daten und neuer Abhängigkeiten.

Ein Mittelweg zwischen der Nutzung globaler ,Clouds' und dem Aufwand zur Einrichtung individueller Arbeitsplätze mit annähernd vergleichbarer Funktionalität sind regional oder sektoral betriebene ,private clouds', die z. B. einen nationalen Bildungssektor unterstützen können. Diese bewähren sich aktuell durchaus hinsichtlich der Bereitstellung spezifischer Inhalte, nicht jedoch als generische Plattformen für Interaktion und Online-Funktionalität im Sinne Cloud-basierter, Software as a Service‘ (,SaaS $\left.{ }^{`}\right)$. 
Aktuelle, zunächst jedoch nicht quantifizierte oder anderweitig bewertete Erfahrungen demonstrieren, dass die Verfügbarkeit und Stabilität der Infrastruktur bei variabler und unerwarteter Belastung bei größeren, d. h. globalen Einheiten, deutlich besser ist. Eine durchaus erwartbare Diagnose.

\section{Von der Techware zur Brainware}

Ausgehend von einer Cloud-zentrierten Infrastruktur können die Anforderungen an lokale Endgeräte-Ausstattung weitgehend reduziert werden, es braucht kaum Computerräume, und die Nutzung von Heimgeräten als vollwertige Zugangspunkte ohne Installations- und Lizenzierungsprobleme ist gegeben. Letztlich brauchen wir „nur“ eine Browser- oder App-Schnittstelle und ausreichende Bandbreite der Verbindung ins Internet.

Sehr wohl jedoch sind adäquate, digitale Kompetenzen' zur effektiven NutzungdieserInfrastrukturerforderlich. Diese können - aufbauend - differenziert werden:

- Plattformkompetenz: Kann ich mit dem Endgerät und generischer Software gewandt umgehen, alltägliche Probleme diagnostizieren und beheben, Apps installieren und Cyber-Angriffe erkennen?

- Medienkompetenz: Kann ich mit allgemeinen Inhalten (z. B. Massenmedien) auch kritisch umge- hen, Inhalte finden, selektieren, interpretieren, weiterleiten etc.?

- Geomedienkompetenz: Kann ich in / mit fach- und domänenspezifische Daten und Medien navigieren, sie interpretieren und auch selbst generieren sowie gezielt zur Vermittlung spezifischer Inhalte einsetzen?

Diese teilweise fachspezifische ,Brainware' als Kombination von Fertigkeiten, Anwendungserfahrung und kontextuellem Wissen ist Voraussetzung, um angeleitet und zunehmend eigenständig arbeiten und lernen zu können. Solange diese Voraussetzung nicht gegeben ist, wird Lernen online nicht oder nur sehr eingeschränkt erfolgreich sein können. Eine verstärkte Betonung des Erwerbs digitaler Kompetenzen (vgl. das digi.komp-Komptenzmodell - https://digikomp.at) gewinnt daher aktuell zusätzliches Gewicht. - Diese können nicht gleichzeitig Voraussetzung und Ergebnis von ,learning-from-home‘ sein.

\section{Soziales Lernumfeld - online?}

Lernen wird in den meisten Fällen im institutionellen Umfeld als soziale Aktivität organisiert. Ein Kritikpunkt an Online-Lernen ist die Reduktion der

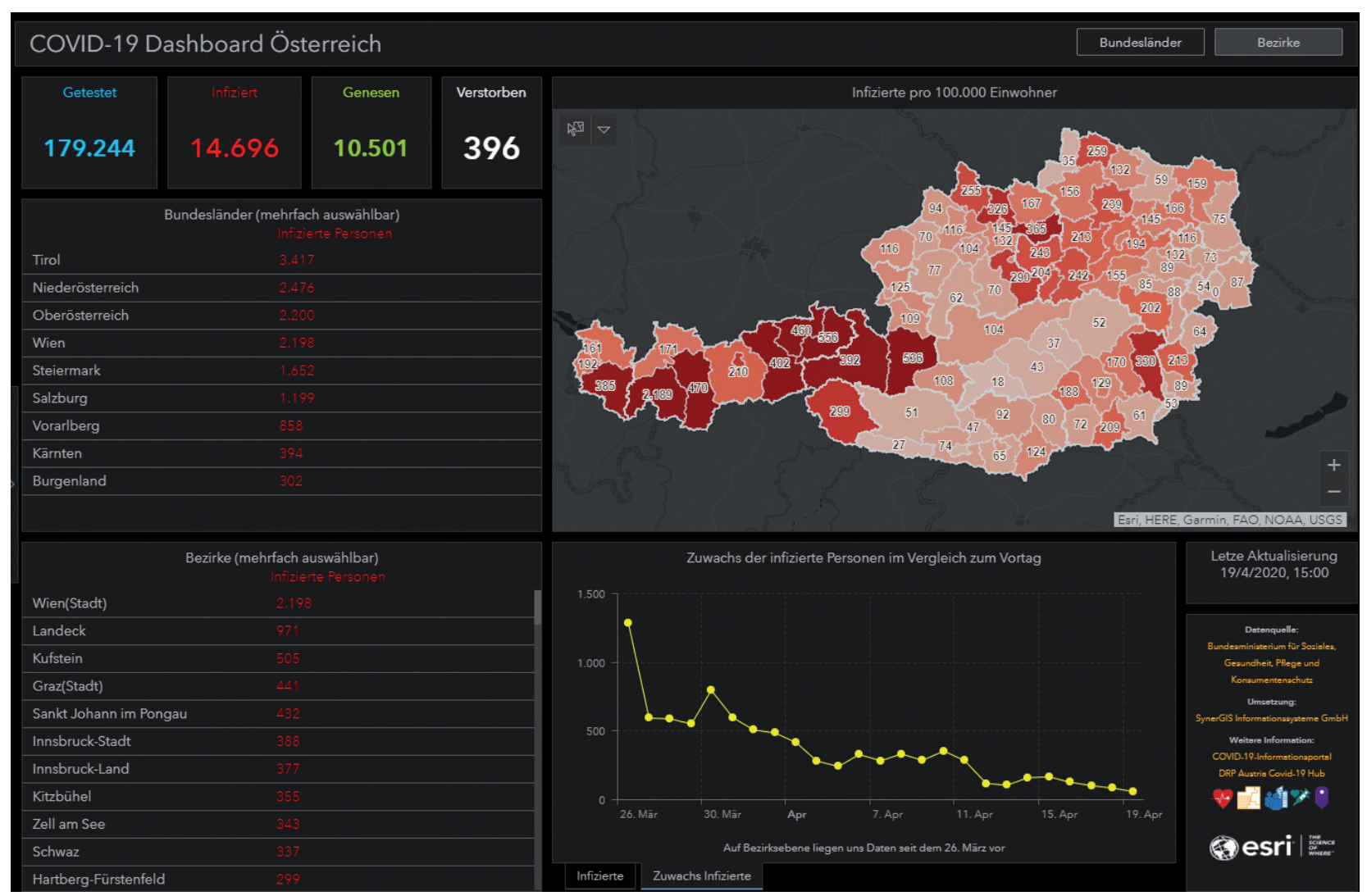

Abb. 1: Corona-Dashboard - Synergis Informationssysteme (2020): Covid-19 Dashboard Österreich vom 19.04.2020. https:/lexperience. arcgis. com/experiencelfb603473e1f74f0bbae48155ff238565 (19.4.2020)

Dashboards prägen mittlerweile unseren alltäglichen Medienkonsum. Die Interpretation der kombinierten Informationen zu Was - Wann - Wo kann mit entsprechend formulierten Aufgabenstellungen zur Entwicklung von Medienkompetenz beitragen. 
Einbindung in ein soziales Umfeld (, was im Übrigen auch den potenziellen Vorteil stärker individualisierbaren Lernens mit sich bringt!).

Davon ausgehend, dass Motivation, Peer Feedback, Beobachten anderer, situatives Mentoring und einfach der persönliche Impuls von Lehrenden wichtige Elemente am Weg zum Lernerfolg sind, kommt der ReKreation des sozialen Kontexts im Cyberspace wohl eine wesentliche, vielleicht sogar DIE zentrale Rolle zu.

Schüler/innen haben mehrheitlich umfassende Erfahrung mit Social Media, d.h. sie sind es durchaus gewohnt, das persönliche soziale Umfeld (auch) online zu gestalten. Aus diesem informellen und egalitären Kontext in eine strukturierte und zielorientierte Situation zu wechseln, ist allerdings offenbar eine neue Herausforderung.

Erfolgreiches Online-Lernen - die Erfahrung des Autors entspringt allerdings vorrangig einem universitären Umfeld - braucht die persönliche Kommunikation sowohl mit Lehrenden wie auch mit anderen Lernenden. Dies zeigen auch die Auswertungen der erfolgreichen Absolvierung von ,Massiv Open Online Courses' (MOOCs) in den letzten Jahren (Robinson et al 2015). Ein wichtiger Schritt im aktuellen Umfeld liegt daher in der ,Professionalisierung' der auf Lernen ausgerichteten Kommunikation.

Dabei ist zwischen synchronen und asynchronen Abläufen ebenso wie zwischen individuell-bilateraler und gruppenbasierter Kommunikation zu differenzieren. Individualisierte, selbstgesteuerte Lernphasen sind gut mit asynchronen Medien, z. B. in Form von Diskussionsplattformen, direkter E-Mail oder mit

\section{Chorus network traffic}

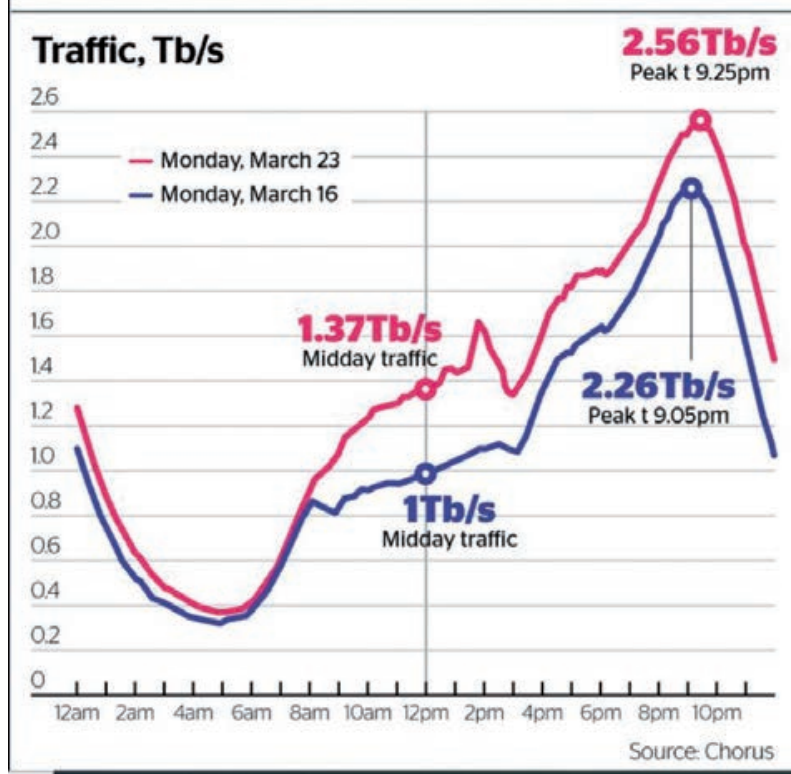

Abb. 2: Tages-Netzauslastung eines neuseeländischen Internet-Providers Eine spannende Interpretationsaufgabe: Wie kommt es zu diesen Veränderungen in der Netzauslastung eines neuseeländischen Internet-Betreibers?
Messaging, zu unterstützen. Dem gegenüber sind synchrone Elemente sowohl für individuelle ad hoc Unterstützung und für die Vermittlung neuer Themen und den sozialen Zusammenhang zu präferieren.

Für die damit verbundene Erzielung von ,Präsenz' ist Live-Video schwer zu ersetzen. Mit einem geschätzt halben Dutzend aktuell populärer Plattformen werden Erfahrungen gesammelt, wobei einige kritische Leistungsmerkmale zu beachten sind:

- Anzahl gleichzeitiger Videostreams - Können sich alle Teilnehmer/innen gleichzeitig sehen?

- Kontrolle über Teilnahme und Wortmeldungen Wie kann ein Austausch flexibel gestaltet werden? - mit ,Handheben', Ansprache von SchülerInnen etc.

- Gruppenarbeit - Können Partner- und Gruppenarbeit nahtlos von und nach Plenarphasen übergeleitet werden?

- Teilen erarbeiteter Dokumente - Wie sind kollaborative Erarbeitung und Annotation bzw. Kommentierung in mündlicher und schriftlicher Form möglich?

Erfahrungsgemäß sind längere Phasen video-basierter Interaktion anstrengend. Einerseits sind sie wichtig für Motivation, Taktung, Vermittlung und Zielkontrolle, und anderseits müssen sie sinnvollerweise mit individuellen Offline-Arbeitsphasen alternieren, um Teilnehmer/innen nicht zu überlasten und mental „bei der Sache“ zu halten.

Kollaborative (peer-to-peer) Arbeit in Gruppen ist, auch ausgehend von bestehenden Erfahrungen mit sozialen Medien, ein zunehmend wichtiger Baustein, dessen nahtlose Einbindung in Unterrichtsabläufe beträchtliche Erfahrung und Fokussierung seitens der Lehrenden braucht. Auch daraus ergibt sich die Überlegung (oder die Forderung?), dass synchrone (,live') Phasen des Unterrichts zwei Personen zur effizienten Umsetzung brauchen. Konzentration auf fachliche Inhalte und persönliche Interaktion einerseits und andererseits auf Moderation und Regie der OnlinePlattform ist nur sehr eingeschränkt von einer Lehrperson machbar.

\section{Vom Notnagel zur regulären Komponente}

Viele Institutionen - auch die Universität - blockierten bis vor Kurzem das nunmehr ubiquitäre und zwingende, work-from-home ' aus unterschiedlichen Gründen. Nach dem aktuellen ,Feldtest' wird das Pendel nicht mehr gänzlich zurückschwingen, zu viele Argumente wurden effektiv ausgeräumt.

Wie wird das mit Online-Lernen sein? Ähnlich wie in der sonstigen Arbeitswelt sind die sozialen, organisatorischen und infrastrukturellen Vorteile einer 


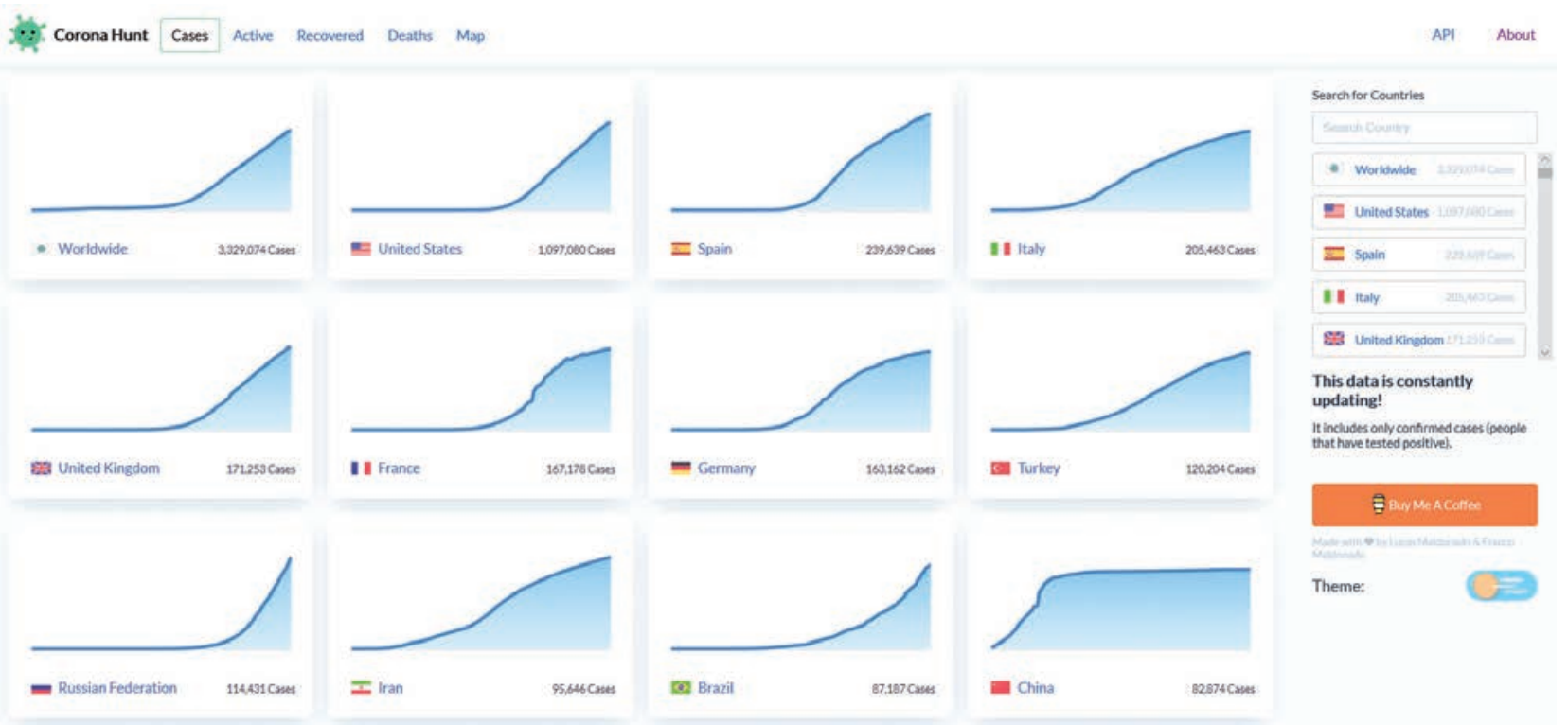

Abb. 3: Corona Hunt-Maldonado, L. \& F. Maldonado (2020): Corona Hunt. https://coronahunt.appl (29.4.2020)

Die Coronahunt.app-Plattform eröffnet analytische und interpretative Zugänge zu zahlreichen Fragestellungen. Das Verstehen globaler Prozesse benötigt insbesondere auch die Berücksichtigung der zeitlichen Dimension!

fokussierten und abgegrenzten Lernumgebung nicht plötzlich verschwunden. Ebenso wie in der Lohnarbeit eine höhere Akzeptanz für z. B. einen Home-Office-Tag zu erwarten ist, sind vermehrte Bausteine in Form von Online-Lernen anzustreben.

Dies nicht nur um für zukünftige Szenarien besser vorbereitet zu sein und die organisatorische Resilienz zu erhöhen, sondern auch, um die spezifischen Vorteile dieses Modus umfassender zu nutzen und um die ,Selbstverständlichkeit' des Agierens in einer zunehmend digital vernetzten Welt als Lernziel zu erreichen.

Daraus ergibt sich die Forderung, Online Lernen in Zukunft nicht nur in Reserve zu halten, sondern auch zu praktizieren. Je nach Altersstufe etwa in Form eines Online-Tages - an diesem kann von daheim, aus einer Betreuungseinrichtung oder aus einem dedizierten Arbeitsraum in den Schule teilgenommen werden.

Dabei geht es nicht nur um Vorbereitet-sein und Einstimmung auf zukünftiges Online-Arbeiten. Die spezifischen Vorteile von Online-Lernen sollen durchaus auch im Normalbetrieb des Unterrichts verstärkt wahrgenommen werden, z. B.

- Verstärkte Individualisierung des Lernens

- Gleichzeitiges und individuelles Feedback

- Direkter Zugang zu Vertiefung und persönlichen Interessen

- Verbindung von Lern-, Alltags- und fachspezifischen Medien

Ein solcher Online-Schultag, hier einfach einmal als Szenario und Anregung postuliert, darf aber eben nicht nur zur digitalen und individuellen Abarbeitung von Arbeitsaufträgen und Aufgaben dienen, sondern muss gleichermaßen die sozialen und kommunikativen Interaktionen beinhalten. Lernen als soziale Online-Aktivität will auch geübt sein.

\section{Fragen für begleitende bzw. nachträgliche Bildungsforschung}

Die aktuelle Situation demonstriert, dass OnlineLernen von daheim - durchgehend von Präsenzunterricht und teilweise auch von synchronen Lernphasen entkoppelt - nicht einfach als unmittelbare Anwendung bisheriger Erfahrungen mit digitalen und Online-Lernmedien realisiert werden kann. Zahlreiche Fragen werden ausgehend davon in nächster Zeit zu adressieren sein:

- Welcher Grad an selbst- (oder peer-) kontrolliertem Wissens- und Kompetenzerwerb entspricht welcher Entwicklungsstufe, welchen Themen und Fächern?

- Für welche Inhalte bzw. Lernziele ist eine virtuelle Kopräsenz (beispielsweise mit Live-Videostream) besonders wichtig bzw. unentbehrlich?

- Welche Rollen, Aufgaben und damit Fertigkeiten sind seitens Lehrender zu erfüllen bzw. einzubringen, um Phasen des Unterrichts zumindest gleichwertig und hoffentlich mit Mehrwert online umzusetzen?

- Für welche wichtigen Elemente erfolgreichen Unterrichts bestehen derzeit keine adäquaten Modelle und Werkzeuge im Cyberspace?

Zahlreiche, auch international und EU-finanzierte Projekte haben über Jahre hinweg versucht, eine ver- 
stärkte Digitalisierung (auch) schulischen Lernens zu ermöglichen. Im Mittelpunkt stand dabei die Generierung von digitalem ,Content' und in zweiter Linie die Entwicklung von Plattformen im Sinne von Learning Management Systems. Die aktuellen Erfahrungen zeigen weniger einen Mangel an ersterem, sehr wohl jedoch Defizite in der Anwendungspraxis dieser Plattformen. Die Distribution digitaler Medien ist als Begleitung zu Präsenzunterricht hilfreich, wenn es jedoch um dessen - auch nur partiellen Ersatz - geht, werden Mängel klar erkennbar.
Deren Behebung werden wir uns in naher Zukunft verstärkt widmen dürfen und müssen!

\section{Literatur}

Robinson, A., J. Kerski, E. Long, H. Luo, D. DiBiase \& A. Lee (2015): Maps and the geospatial revolution: teaching a massive open online course (MOOC) in geography. Journal of Geography in Higher Education 39(1). S. 65-82. https://doi.org/10.1080/03098265.20 14.996850 